\title{
Influence of an upstream building on the wind-induced mean suction on the flat roof of a low-rise building
}

\author{
Santiago Pindado, José Meseguer, Sebastián Franchini*
}

\begin{abstract}
A B S T R A C T
The effect of an upstream building on the suction forces on the flat roof of a low-rise building placed in the wake of the former is analyzed. The analysis has been performed by wind tunnel testing of a flat roof, low-rise building model equipped with pressure taps on the roof and different block-type buildings (only configurations where the upstream building is as high or higher than the downstream one are considered in this paper). The influence of the distance between both buildings on the wind loads on the downstream building roof is analyzed, as well as the height of the upstream one and the wind angle of incidence. Experimental results reveal that the wind load increases as the relative height of the upstream building increases, the wind load being highest for intermediate distances between buildings, when a passage between them is formed.
\end{abstract}

\section{Introduction}

As it is well known and documented, the largest wind loads produced on the roofs of buildings are caused by the vortex flow pattern generated on the surface of the roof (Banks et al., 2000; Banks and Meroney, 2001a, 2001b; Franchini et al., 2005; Hoxey et al., 1998; Kawai, 1997, 2002; Lin et al., 1995; Marwood and Wood, 1997; Wu et al., 2001a, 2001b). This vortex flow pattern is generated close to the roofs' corners for oblique wind directions and it normally consists of two conical vortices, each associated to one of the two edges that form the roof corner (Fig. 1).

Commonly, complete scale models of urban areas are needed for the wind tunnel tests performed to study the wind effects on a single building. Such tests give accurate information about the pressure distribution on building façades and roofs, taking into account that upstream buildings can accelerate the flow, thereby increasing the suction loads on the surfaces of the building under consideration. However, in spite of the large amount of information concerning wind loads on isolated low-rise buildings, there does not seem to be much data concerning the effect of upstream obstacles on the conical vortices generated on the roof of a downstream building.

These upstream obstacles modify both the velocity distribution and the turbulence level of the wind flow in the surroundings of the downstream buildings, so it is reasonable to expect changes in the pressure distribution on them compared to that on an isolated building in a free stream. In this sense, research done in the past has pointed out the influence of adjacent buildings on the wind-induced loads on buildings, either low or tall. Some authors have focused their studies on local wind loads on roofs and façades (Ahmad and Kumar, 2001; Case and Isyumov, 1998; Ho et al., 1990; Jóźwiak et al., 1995; Khanduri et al., 1998, Lam et al., 2008, 2009), whereas some others have focused on the overall structural loads on buildings, including the changes on the windinduced dynamic response of tall buildings due to other upstream buildings (Khanduri et al., 1998; Lia et al., 2006; Niemann and Köpper, 1998; Tang and Kwok, 2004; Thepmongkorn and Kwok, 2002; Thepmongkorn et al., 2002; Xie and Gu, 2007; Zhang et al., 1995). The influence of the urban environment normally reduces the suction loads on the low-buildings as they are embedded in the surroundings (Case and Isyumov, 1998). However, in the case of two buildings (or a less dense configuration of buildings) an increase of the wind suctions has been also pointed out (Ahmad and Kumar, 2001; Jóźwiak et al., 1995).

Besides, the effect of the wind angle of incidence, $\beta$ (Fig. 1), on the roof of low-rise buildings placed in a free stream has been widely studied. The suction loads on a roof become a maximum only for certain oblique wind directions, normally close to $\beta=45^{\circ}$ (Kawai, 2002), provided the building is symmetrical in relation to such wind direction. However, it must be pointed out that depending on the size of the building and the shape of the roof (canopy, gable, etc.) the wind direction, which produces the largest suction load can be quite different from $\beta=45^{\circ}$ (Franchini et al., 2005; Hoxey et al., 1998; Lin et al., 1995; Wu et al., 2001a).

The aim of this communication is to give some insights into the effect of an upstream building on the mean suction forces on the flat roof of a second building. The influence of the distance 

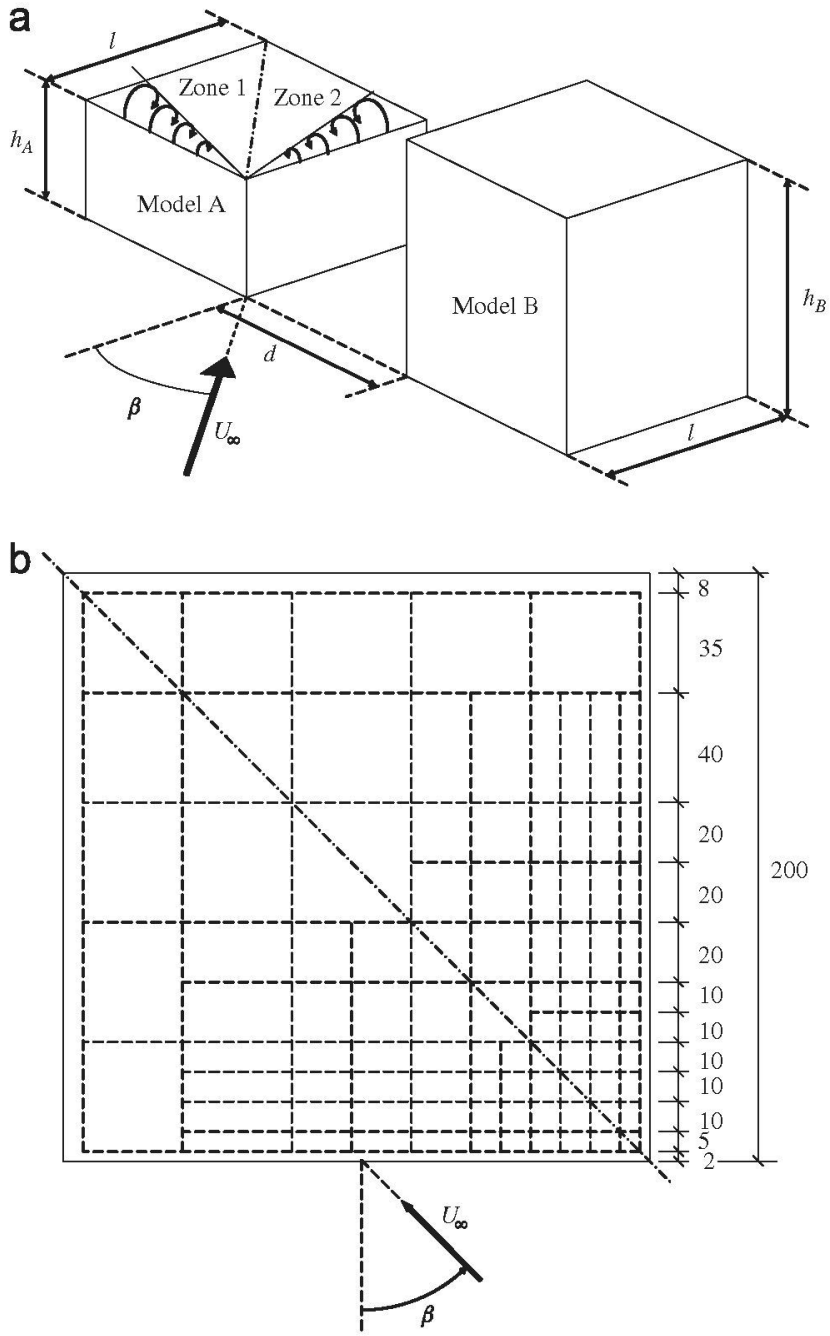

Fig. 1. (a) Sketch of the two-building configuration studied. The wind direction angle, $\beta$, the two zones (Zone 1 and Zone 2 ) into which the roof of the model $A$ has been divided are indicated, as well as the conical vortices formed on the roof of this model in case of an oblique free stream (without the influence of the upstream model B); (b) sketch of the pressure taps position on the roof of the model A (dimensions are in $\mathrm{mm}$ ).

between both buildings and the height of the upstream one on the wind loads on the downstream building roof is investigated through wind tunnel experiments. Only configurations where the upstream building is as high as or higher than the downstream one are considered. The analysis has been performed using the measured mean pressure distributions on the flat roof of a lowrise building for different building configurations obtained by changing both, the height of the upstream building and the separation between buildings.

The use of mean pressure distribution is because, as is well known, the correlation between wind tunnel simulation and fullscale wind loads is higher in terms of average pressures, but not in terms of peak pressures, since they strongly depends on the turbulence intensity in longitudinal and lateral directions and the power spectrum density of the free stream (Tieleman, 1996, 2003).

\section{Experimental configuration}

The configuration under study, as sketched in Fig. 1, consists of two different box-shaped building models, placed at a distance $d$ from one another. Both models have the same side length $(l=0.2 \mathrm{~m})$. The height of the model A (the one equipped with pressure taps on the flat roof) is $h_{\mathrm{A}}=0.1 \mathrm{~m}$, whereas for the model $\mathrm{B}$ four different heights were considered $\left(h_{\mathrm{B}} / h_{\mathrm{A}}=1,1.5,2\right.$ and 3$)$. In order to compare the wind loads measured on model $A$ roof to that measured without the influence of any upstream building, an isolated configuration of model A was also analyzed in the testing campaign (this case will be denoted as $h_{\mathrm{B}} / h_{\mathrm{A}}=0$ hereinafter). Six different distances between both models were considered for each height of the model $\mathrm{B}\left(d / h_{\mathrm{A}}=0,0.5,1,2,3\right.$ and 4$)$. Each configuration was measured for twelve different wind directions, $\beta=0^{\circ}, 15^{\circ}, 25^{\circ}, 30^{\circ}, 35^{\circ}, 40^{\circ}, 45^{\circ}, 50^{\circ}, 55^{\circ}, 60^{\circ}, 75^{\circ}$ and $90^{\circ}$.

The distribution of pressure taps on the roof of the model $A$ is shown in Fig. 1. There are 116 pressure taps on the roof, their density increasing close to the windward sides of the roof, where the larger pressure variations are expected. Each pressure tap consists of a $1 \mathrm{~mm}$ inner diameter brass tube, which is connected to the pressure measurement instrument by a plastic tube with $1 \mathrm{~mm}$ inner diameter. The plastic tubes are connected to two 64-connection pressure scanners from Scanivalve Corp. (ZOC 33). 2000 samples were taken in over $10 \mathrm{~s}$ in each pressure tap throughout the measurements. The pressure coefficient is defined as $c_{p}=\left(p-p_{\infty}\right) / q_{\infty}$, where $p$ is the average pressure measured on each tap, and $p_{\infty}$ and $q_{\infty}$ are the static and dynamic pressures measured upstream of both building models and at model A's roof height.

Measurements were carried out in the A9 Wind Tunnel at the IDR/UPM, where the test chamber has a width of $1.5 \mathrm{~m}$ and a height of $1.8 \mathrm{~m}$. The wind velocity profile at the model test section was similar to type I atmospheric boundary layer distribution (as defined in Eurocode 1), the turbulence intensity at the model A roof height being around $4 \%$. It must be remarked that the lowturbulence testing condition can be considered a more severe condition than turbulent flow (Kawai, 1997; Suzuki et al., 2003; Tieleman, 2003), and the correlation with full-scale measurements is better when comparing average wind loads than when comparing peak wind loads (Ahmad and Kumar, 2001; Ho et al., 2005). No blockage corrections of the measured results has been considered, as even in the worst case the normal-to-wind areas of the testing models were smaller than $8 \%$ of the wind tunnel crosssection. The wind velocity of the stream at the test section of the wind tunnel, at the model $\mathrm{A}$ roof height, was close to $25 \mathrm{~m} \mathrm{~s}^{-1}$.

\section{Results and discussion}

The roof of model $\mathrm{A}$ has been divided in two zones, named Zone 1 and Zone 2, in order to separately study the areas where each conical vortex is formed (Fig. 1). As expected, very high suctions have been measured in both zones with model $A$ standing alone in an oblique free stream (without the model B placed upstream). The maximum suction in each zone being higher than $-c_{p}=5.5$. Those higher values of suction were measured at different wind angles, $\beta=40^{\circ}$ in Zone 1 and at $\beta=50^{\circ}$ in Zone 2.

Because of the huge amount of information gathered during this wind tunnel testing, the results are presented as follows: first of all some representative pressure distributions on model A's roof are shown (Figs. 2-4). In Fig. 5 the maximum suction loads measured on the model $A$ are plotted as a function of the wind angle of incidence $\beta$ and different configurations. It is important to remark that the pressure coefficients plotted in Fig. 5 are the maximum values obtained from the mean pressure distribution on model A roof.

Note that the case of isolated building $A$ can be considered as the limiting case of either $h_{\mathrm{B}} / h_{\mathrm{A}} \rightarrow 0$ or $d / h_{\mathrm{A}} \rightarrow \infty$.

In Fig. 2 pressure coefficient contours on the roof of model $A$ are shown for different $h_{\mathrm{B}} / h_{\mathrm{A}}$ ratios and wind angles of incidence. 


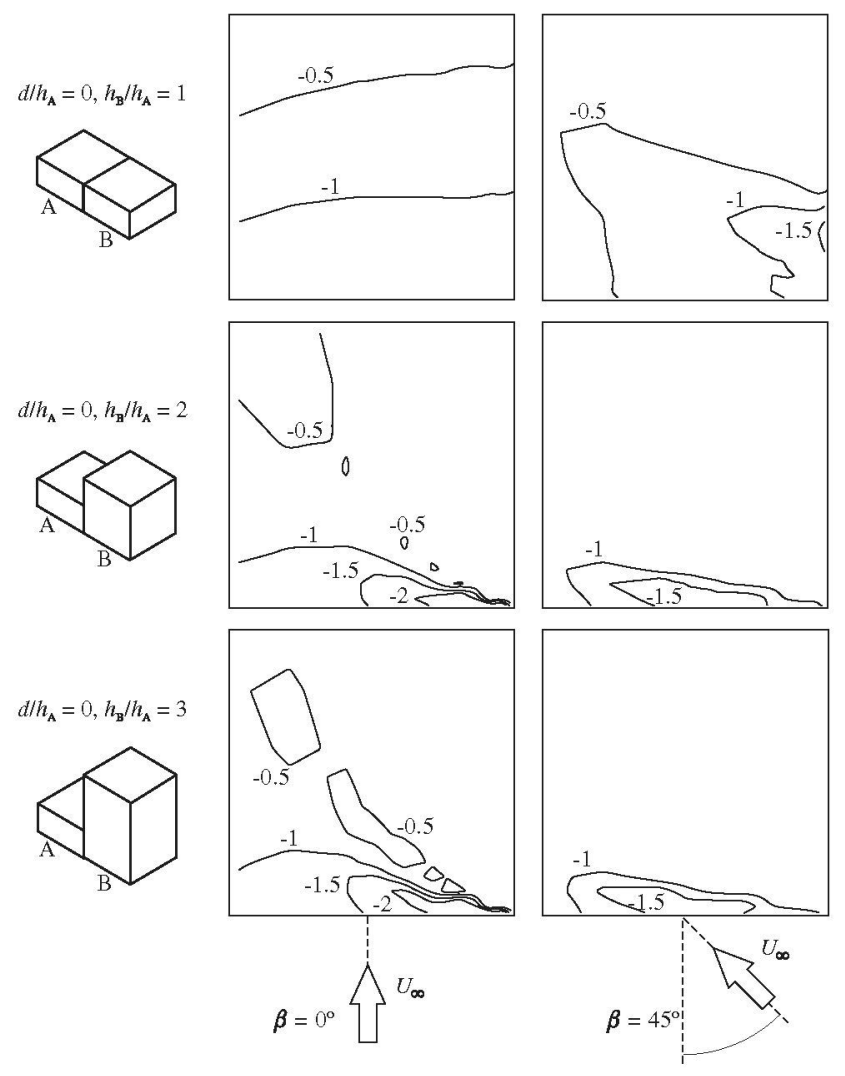

Fig. 2. Pressure coefficient distributions on the model A roof for different upstream model heights, $h_{\mathrm{B}} / h_{\mathrm{A}}$, and wind directions $\beta$. The results correspond to a dimensionless distance between models $d / h_{A}=0$. Labels on the graphs indicate the values of the pressure coefficient.
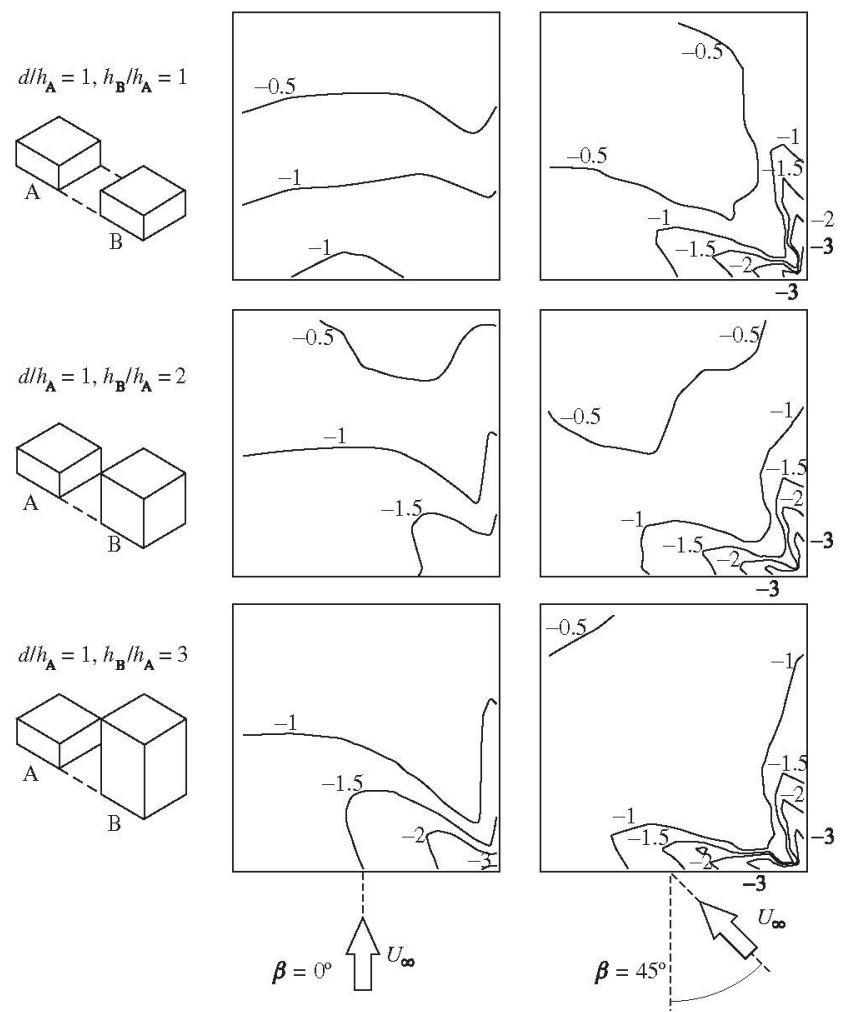

Fig. 3. Pressure coefficient distributions on the model A roof for different upstream model heights, $h_{\mathrm{B}} / h_{\mathrm{A}}$, and wind directions $\beta$. The results correspond to a dimensionless distance between models $d / h_{\mathrm{A}}=1$. Labels on the graphs indicate the values of the pressure coefficient.
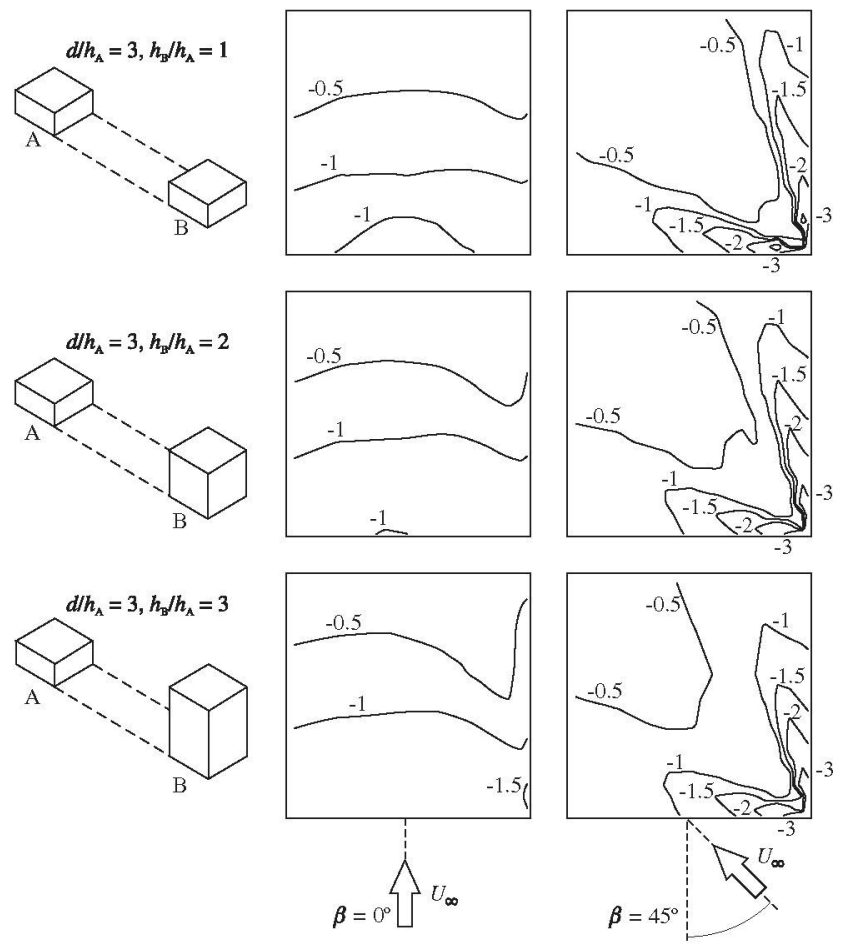

Fig. 4. Pressure coefficient distributions on the model $A$ roof for different upstream model heights, $h_{\mathrm{B}} / h_{\mathrm{A}}$, and wind directions $\beta$. The results correspond to a dimensionless distance between models $d / h_{A}=3$. Labels on the graphs indicate the values of the pressure coefficient.

In these cases, both models are placed one next to the other, $d / h_{\mathrm{A}}=0$, so they can be considered as a single building. According to the different sketches (see also Fig. 5), the most critical situation seems to take place when the wind direction is $\beta=0^{\circ}$ and model $B$ is higher than model $A$. The reason being that wind is forced by model B to change its direction over the model A roof, generating a conical vortex anchored to the upstream edge of the latter's roof (that is to say, over Zone 1).

When $\beta=90^{\circ}$ and both buildings have the same height, model A's roof can be considered as the rear part of the common roof, so no significant increase in wind pressures due to conical vortices can be reported. As the height of model B grows, model A's roof becomes more and more in the wake of the former building, therefore pressures on this roof are mainly driven by the flow separation on model B. For oblique wind directions such as $\beta=45^{\circ}$, the contours pattern shows a conical vortex starting at the corner where the roof of model A meets model B provided $h_{\mathrm{B}} / h_{\mathrm{A}}>1$, or to the conical vortex formed at the roof of model $\mathrm{B}$ windward corner when $h_{\mathrm{B}} / h_{\mathrm{A}}=1$.

Pressure distributions on the roof of model A drastically change when there is a gap between both models, as can be observed in Fig. $3\left(d / h_{\mathrm{A}}=1\right)$ and in Fig. $4\left(d / h_{\mathrm{A}}=3\right)$. In both cases, intense conical vortices are formed for oblique winds directions, the same pattern being observed on Zone 1 of building A's roof for small values of the angle of incidence provided both models are close enough. The air passing through the passage between the models modifies the local flow over Zone 2 when model $B$ is higher than the model $\mathrm{A}$. In these cases, the suction is increased close to the roof edge of the aforementioned zone for small wind angles, $\beta \approx 0^{\circ}$, and the well known conical vortex pressure distribution pattern also being modified in this zone for oblique wind directions. For small wind angles and a small separation between models (Fig. $3, d / h_{\mathrm{A}}=1$ ) the suction on Zone 1 is mainly the same as the one with no gap between them, that is, the wake downstream a taller model $\mathrm{B}\left(h_{\mathrm{B}} / h_{\mathrm{A}}>1\right)$, creates a conical vortex 

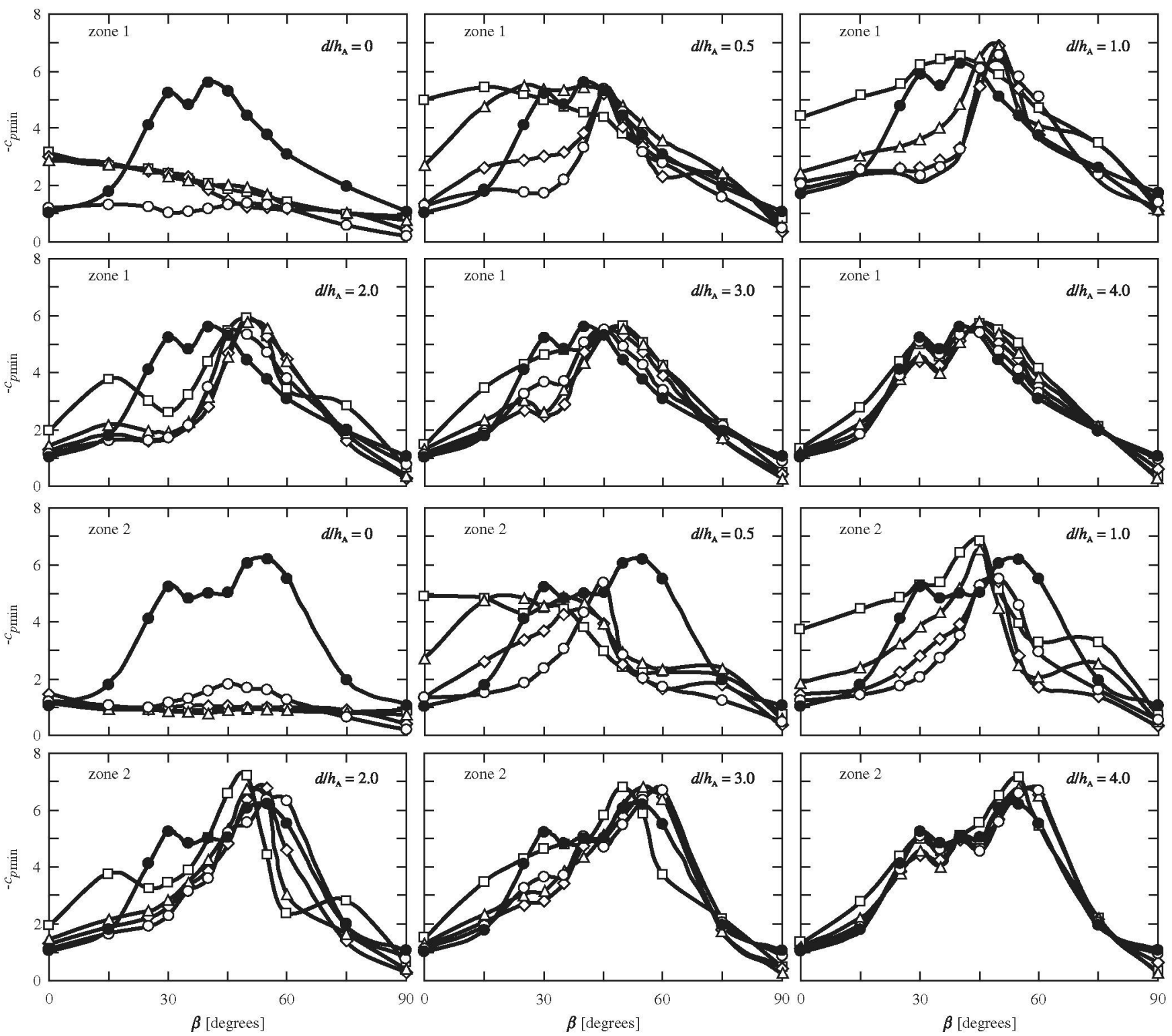

Fig. 5. Variation with the wind direction, $\beta$, of the minimum pressure coefficient, $c_{p m i n}$, measured both in Zone 1 and in Zone 2 for different values of the dimensionless distance $d / h_{\mathrm{A}}$ (as indicated in the inserts). The symbols identify the relative height of model B according to the following key: $h_{\mathrm{B}} / h_{\mathrm{A}}=0$ (closed circles), $h_{\mathrm{B}} / h_{\mathrm{A}}=1$ (circles), $h_{\mathrm{B}} / h_{\mathrm{A}}=1.5$ (rhombi), $h_{\mathrm{B}} / h_{\mathrm{A}}=2$ (triangles) and $h_{\mathrm{B}} / h_{\mathrm{A}}=3$ (squares).

pattern suction on this area of the roof. On the other hand, for the oblique wind directions the air flow passing through the passage interacts with the conical vortex formed over Zone 2 , increasing the local velocity of the vortex in the direction of its axis and enlarging the suction area on Zone 2 as the vortex breakdown is now produced further from the vortex origin. This passage effect, which can be observed in Fig. 4, tends to disappear as the distance between both models grows (Fig. 5).

The maximum suction loads, $-c_{p \text { min }}$, appearing both in Zone 1 and in Zone 2 for each value of the wind angle of incidence $\beta$ and different model configurations are plotted in Fig. 5. In all plots, together with the data corresponding to the different relative heights, $h_{\mathrm{B}} / h_{\mathrm{A}}$, the curve $h_{\mathrm{B}} / h_{\mathrm{A}}=0$ (model $\mathrm{A}$ isolated or $d / h_{\mathrm{A}} \rightarrow \infty$ ), has also been represented.

According to Fig. 5 both Zone 1 and Zone 2 behave in a similar way, although suctions measured in Zone 2 are more severe than in Zone 1. This is explained because of the interference between buildings, although such explanation does not hold in the case of model $\mathrm{A}$ isolated $\left(h_{\mathrm{B}} / h_{\mathrm{A}}=0\right)$. In the isolated building case the discrepancy between both zones can be explained because of small differences in the sharpness of windward roof edges, together with little differences in the position of symmetrical taps $( \pm 0.5 \mathrm{~mm})$ and uncertainties in the angular position of the test model with respect to the incident flow, which is estimated to be $\pm 0.5^{\circ}$ in this measurement series.

Leaving aside the above comments, it is clear from Fig. 5 that the influence of the upstream building becomes negligible once the distance between buildings becomes four times or larger than model $A$ height $\left(d / h_{\mathrm{A}} \geq 4\right)$, irrespective of the value of the wind angle of incidence. For intermediate distances, $1 \leq d / h_{\mathrm{A}} \leq 4$, the presence of the upstream building can produce larger suction loads than the ones corresponding to the isolated building case. Relative load increments can be dramatic at low values of the angle of incidence (up to nearly $400 \%$ in Zone 1 when $d / h_{\mathrm{A}}=0.5$ and $\beta=0^{\circ}$ ), although the suction loads at these low values of the angle of incidence are smaller than the highest ones measured in free stream. For oblique winds, $\beta \approx 45^{\circ}$, relative load increments are not so dramatic, though it must be remarked that suction 
loads can become larger than those measured in the isolated building.

The influence of an upstream building on the wind loads is commonly expressed in terms of the interference factor, $I F$, defined as $I F=P_{\text {interference }} / P_{\text {isolated }}$, where $P_{\text {interference }}$ is the pressure (other load effects can be also selected) on a building with interference effects, and $P_{\text {isolated }}$ is the pressure on the same building but isolated (Ahmad and Kumar, 2001; Khanduri et al., 1998). The maximum value of the interference factor for the configuration under study is $I F=1.21$, although it must be remarked that a quite large interference factor (taking into account average loads), $I F=1.41$, has been measured in wind tunnel on a low-rise hip roof building when a similar building was located upstream of it (Ahmad and Kumar, 2001).

\section{Conclusions}

The influence of an upstream building on the wind loads on the flat roof of a low-rise building has been analyzed by wind tunnel tests of appropriate models. Different values of the ratio of the height of the upstream building to the height of the downstream building (the one equipped with pressure taps) were considered, as well as different separation distances between them.

Experimental results show that wind loads on the flat roof of a low-rise building can be magnified by the presence of an upstream building higher than the one under consideration. The wind load increases as the relative height of the upstream building increases, and it becomes maximum for intermediate distances $\left(d / h_{\mathrm{A}} \approx 1\right)$, when a passage between buildings is formed.

\section{References}

Ahmad, S., Kumar, K., 2001. Interference effects on wind loads on low-rise hip roof buildings. Engineering Structures 23, 1577-1589.

Banks, D., Meroney, R.N., 2001a. A model of roof-top surface pressures produced by conical vortices: Evaluation and implications. Wind and Structures 4, 279-298.

Banks, D., Meroney, R.N., 2001b. The applicability of quasi-steady theory to pressure statistics beneath roof-top vortices. Journal of Wind Engineering and Industrial Aerodynamics 89, 569-598.

Banks, D., Meroney, R.N., Sarkar, P.P., Zhao, Z., Wu, F., 2000. Flow visualization of conical vortices on flat roofs with simultaneous surface pressure measurement. Journal of Wind Engineering and Industrial Aerodynamics 84, 65-85.

Case, P.C., Isyumov, N., 1998. Wind loads on low buildings with 4:12 gable roofs in open country and suburban exposures. Journal of Wind Engineering and Industrial Aerodynamics 77\&78, 107-118.

Franchini, S., Pindado, S., Meseguer, J., Sanz-Andrés, A., 2005. A parametric, experimental analysis of conical vortices on curved roofs of low-rise buildings. Journal of Wind Engineering and Industrial Aerodynamics 93, 639-650.

Ho, T.C.E., Surry, D., Davenport, A.G., 1990. The variability of low building wind loads due to surrounding obstructions. Journal of Wind Engineering and Industrial Aerodynamics 36, 161-170.
Ho, T.C.E., Surry, D., Morrish, D., Kopp, G.A., 2005. The UWO contribution to the NIST aerodynamic database for wind loads on low buildings: Part 1. Archiving format and basic aerodynamic data. Journal of Wind Engineering and Industrial Aerodynamics 93, 1-30.

Hoxey, R., Robertson, A., Short, L., 1998. The role of corner vortices in the design of structures. Structural Engineering International 1/98, 50-55.

Jóźwiak, R., Kacprzyk, J., Zuraski, J.A., 1995. Wind tunnel investigations of interference effects on pressure distribution on a building. Journal of Wind Engineering and Industrial Aerodynamics 57, 159-166.

Kawai, H., 1997. Structure of conical vortices related with suction fluctuation on a flat roof in oblique smooth and turbulent flows. Journal of Wind Engineering and Industrial Aerodynamics 69-71, 579-588.

Kawai, H., 2002. Local peak pressure and conical vortex on building. Journal of Wind Engineering and Industrial Aerodynamics 90, 251-263.

Khanduri, A.C., Stathopoulos, T., Bédard, C., 1998. Wind-induced interference effects on buildings - a review of the state-of-the-art. Engineering Structures $20,617-630$

Lam, K.M., Leung, M.Y.H., Zhao, J.G., 2008. Interference effects on wind loading of a row of closely spaced tall buildings. Journal of Wind Engineering and Industrial Aerodynamics $96,562-583$

Lam, K.M., Leung, M.Y.H., Zhao, J.G., 2009. Erratum to "Interference effects on wind loading of a row of closely spaced tall buildings". Journal of Wind Engineering and Industrial Aerodynamics 97, 110.

Lia, Q.S., Fu, J.Y., Xiao, Y.Q., Li, Z.N., Ni, Z.H., Xie, Z.N., Gu, M., 2006. Wind tunnel and full-scale study of wind effects on China's tallest building. Engineering Structures 28, 1745-1758.

Lin, J.X., Surry, D., Tieleman, H.W., 1995. The distribution of pressure near roof corners of flat roof low buildings. Journal of Wind Engineering and Industrial Aerodynamics $56,235-265$.

Marwood, R., Wood, C.J., 1997. Conical vortex movement and its effect on roof pressures. Journal of Wind Engineering and Industrial Aerodynamics 69-71, 589-595.

Niemann, H.J., Köpper, H.D., 1998. Influence of adjacent buildings on wind effects on cooling towers. Engineering Structures 20, 874-880.

Suzuki, M., Tanemoto, K., Maeda, T., 2003. Aerodynamic characteristics of train/ vehicles under cross winds. Journal of Wind Engineering and Industrial Aerodynamics $91,209-218$

Tang, U.F., Kwok, K.C.S., 2004. Interference excitation mechanisms on a 3DOF aeroelastic CAARC building model. Journal of Wind Engineering and Industrial Aerodynamics 92, 1299-1314.

Thepmongkorn, S., Kwok, K.C.S., 2002. Wind-induced responses of tall buildings experiencing complex motion. Journal of Wind Engineering and Industrial Aerodynamics $90,515-526$

Thepmongkorn, S., Wood, G.S., Kwok, K.C.S., 2002. Interference effects on windinduced coupled motion of a tall building. Journal of Wind Engineering and Industrial Aerodynamics 90, 1807-1815

Tieleman, H.W., 1996. Model/full scale comparison of pressures on the roof of the TTU experimental building. Journal of Wind Engineering and Industrial Aerodynamics $65,133-142$

Tieleman, H.W., 2003. Wind tunnel simulation of wind loading on low rise structures: A review. Journal of Wind Engineering and Industrial Aerodynamics $91,1627-1649$.

Wu, F., Sarkar, P.P., Metha, K.C., 2001a. Full-scale study of conical vortices and roof corner pressures. Wind and Structures 4, 131-146.

Wu, F., Sarkar, P.P., Metha, K.C., Zhao, Z., 2001b. Influence of incident wind turbulence on fluctuations near flat-roof corners. Journal of Wind Engineering and Industrial Aerodynamics $89,403-420$.

Xie, Z.N., Gu, M., 2007. Simplified formulas for evaluation of wind-induced interference effects among three tall buildings. Journal of Wind Engineering and Industrial Aerodynamics 95, 31-52.

Zhang, W.J., Xu, Y.L., Kwok, K.C.S., 1995. Interference effects on aeroelastic torsional response of structurally asymmetric tall buildings. Journal of Wind Engineering and Industrial Aerodynamics 57, 41-61. 